\title{
Technical Papers
}

\section{Analysis and design of trussed structures made of elastic/stiffening materials}

\author{
J.E. Taylor and P.D. Washabaugh \\ University of Michigan, Department of Aerospace Engineering, Ann Arbor, Michigan 48109-2118, USA
}

\begin{abstract}
An extremum problem formulation for the equilibrium analysis of general structures made of stiffening material is applied to the analysis of trussed structures. The nonlinear material is modelled in a way to simulate an arbitrary, polygonal stress-strain relation; material properties may vary over the truss. The form of this convex nonlinear programming problem statement is convenient for the prediction of the evolution (over the truss system) of local member stiffening under increasing proportional load. Computational solutions are obtained directly on the basis of the extremum problem statement, using commercial minimizer software. The formulation for analysis is extended to model a design problem for the prediction of the optimal modification of certain material properties, namely the bounds that reflect the onset of stiffening in the material. Computational results for this optimal material design problem are also provided.
\end{abstract}

\section{Introduction}

The goal of this paper is to demonstrate the utility of a recently developed extremum principle (Taylor 1992) in the context of the analysis and design of structures that include nonlinear-stiffening materials. Briefly, the formulation treats total strain in the nonlinear problem as a superposition of an arbitrary number of independent constituent fields. The problem is expressed in "mixed form" and it has the structure of a convex, constrained, nonlinear extremum problem statement. This additive decomposition of a nonlinear material response is distinct and offers certain advantages over typical formulations (e.g. Hlavacek et al. 1992; Simo et al. 1989; Comi et al. 1991). The model is general for materials of stiffening type; i.e. with the proper identification of a set of parameters, it is possible to simulate any stiffening material. Also, the parameters that are used to define the material properties (here the moduli of each constituent field and bounds on the constituent response) appear explicitly in the problem formulation, and this facilitates an extension of the formulation to treat certain aspects of the design of material properties.

The first aspect of the paper specializes the general principle for its application to the analysis of truss structures made of stiffening materials. The problem formulation is stated and interpreted for general trussed structures. Using a commercially available optimizer package, numerical solutions of two nonlinear trusses are provided as examples of the feasibility and utility of this approach. In the second phase of this study, a convex extremum problem statement is presented for the optimal design of material parameters related to the onset of stiffening. This second problem allows the nonlinear aspects of the structural response to be fashioned within a resource constraint. A numerical example of this material design problem is also provided.

\section{A minimum principle for equilibrium analysis}

The minimum principle described by Taylor (1992) for general continuum structures is specialized here for arbitrary two- or three-dimensional trusses. Our approach is to first describe the structure of the model for the nonlinear stiffening material itself, and then to present the extremum problem statement covering global equilibrium analysis for the truss made of such material. Computational results showing the evolution of hardening over the truss system under increasing load are presented in this section as well.

\subsection{Model description}

Total strain $\varepsilon_{T i}$ of the nonlinear stiffening material is represented (for the $i$-th truss member) in terms of independent measures $\sigma_{i}$ and $\varepsilon_{i}^{\beta}\left(\beta=1,2, \ldots, N_{i}\right)$ as

$\varepsilon_{T i}=\frac{\sigma_{i}}{\tilde{E}_{i}}+\sum_{\beta=1}^{N_{i}} \varepsilon_{i}^{\beta}$.

This superposition corresponds in form to the representation of kinematics for a system in which mechanical elements, each associated with the separate measures in (1), are connected in series; $\tilde{E}_{i}$ represents a specified constant which can be interpreted as the modulus of the $\sigma_{i}$ constituent. Suppose now that the quantities $\varepsilon_{i}^{\beta}$, which are referred to as constituent strains, have the following form:

$\varepsilon_{i}^{\beta}= \begin{cases}\sigma_{i} / E_{i}^{\beta} & \text { when }\left(-\underline{\varepsilon}_{i}^{\beta}<\varepsilon_{i}^{\beta}<\bar{\varepsilon}_{i}^{\beta}\right), \text { else } \\ \bar{\varepsilon}_{i}^{\beta} & \text { when }\left(\sigma_{i} \geq E_{i}^{\beta} \bar{\varepsilon}_{i}^{\beta}\right) \\ -\underline{\varepsilon}_{i}^{\beta} & \text { when }\left(\sigma_{i} \leq-E_{i}^{\beta} \underline{\varepsilon}_{i}^{\beta}\right) .\end{cases}$

Here, values $E_{i}^{\beta}, \underline{\varepsilon}_{i}^{\beta}$, and $\bar{\varepsilon}_{i}^{\beta}$ are specified and can be interpreted as the modulus, lower bound, and upper bound of the $\varepsilon_{i}^{\beta}$ constituent, respectively. This stress-strain relation describes what has been labelled an elastic/locking material (see Fig. 1); the model was first presented by Prager (1957). With this definition of constituent strains $\varepsilon_{i}^{\beta}$, the total strain of (1) has the form shown in Fig. 2. Given that the values specified for parameters $\tilde{E}_{i}, E_{i}^{\beta}, \underline{\varepsilon}_{i}^{\beta}, \bar{\varepsilon}_{i}^{\beta}$, and $N_{i}$, are arbitrary, the polygonal form defined by (1) and (2) is regarded 
to be the general representation of constitutive properties for the stiffening material considered in this paper (we do not address here the identification problem, essentially one of curvefitting, of matching the present model to data for a specific material).

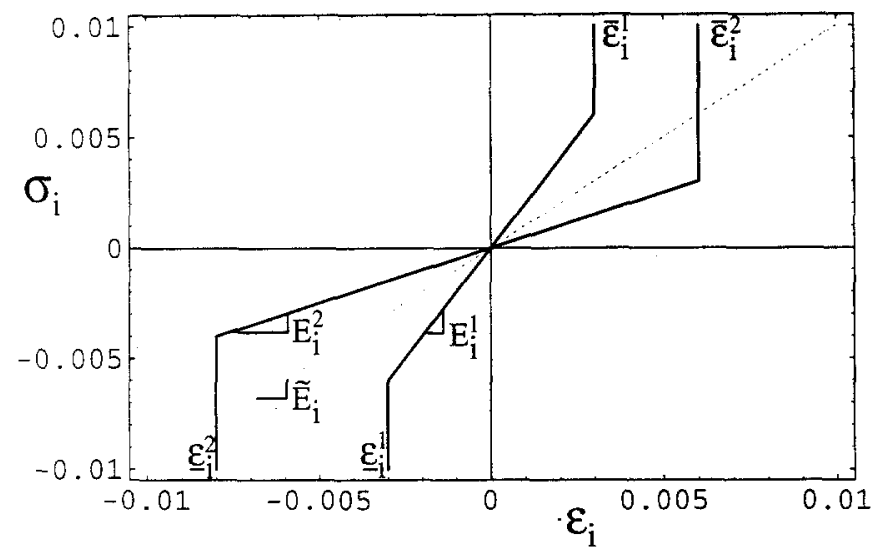

Fig. 1. Plot of the locking and enduring constituent stress strain behaviour of a truss member

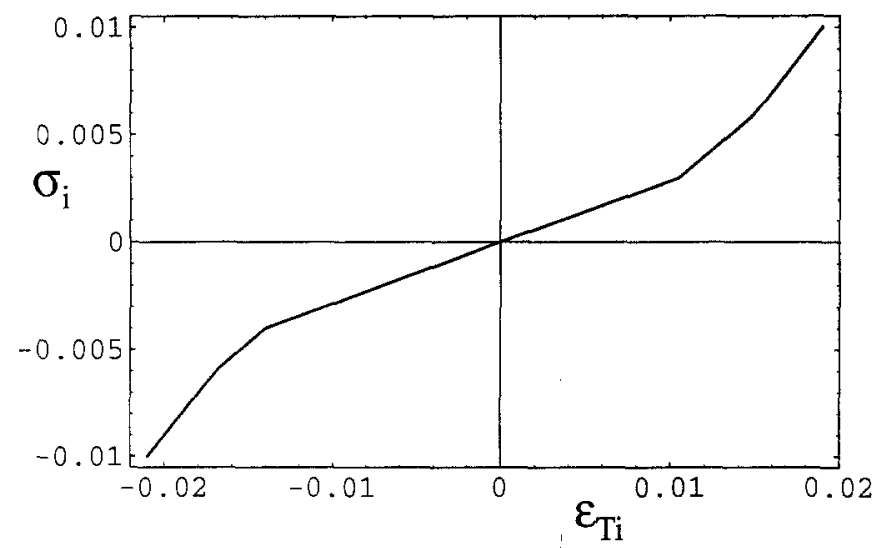

Fig. 2. Plot of the total stress strain behaviour of a truss member

The formulation for global equilibrium analysis is described next. It is distinguished from certain known mixed models for the analysis problem (see e.g. Washizu 1982; Oden and Reddy 1976; Arthurs 1980; Sewell 1987) by the features that it has the form of an extremum problem, and it provides in its structure for specification of material properties (for the general polygonal material) in terms of a set of parameters. We note also that in the present formulation the model for our nonlinear material is incorporated quite simply (in implicit form) within the variational problem statement, and consequently it becomes unnecessary ever to deal directly with the mathematically cumbersome forms of (1) and (2).

The variational problem statement described here can be identified as a generalization of the classical minimum potential energy formulation for equilibrium analysis of systems with linear materials (indeed, the conventional form is recovered as a special case within our formulation) (Taylor 1992). Expressed in words the problem is stated: "minimize a load factor within constraints that bound the value of a generalized potential energy, that reflect limits on the magnitude of the strain constituents introduced in (1) above, and that enforce strain-displacement relations". In the context of the variational problem, the load vector $\alpha$, stresses $\sigma_{i}$, strain constituents $\varepsilon_{i}^{\beta}$, and nodal displacements $u_{\gamma}$ are independent. The problem formulation may be expressed in the following form:

$$
[\mathbf{P}]\left\{\begin{array}{l}
\min _{\alpha, \sigma, \varepsilon, u} \alpha \\
\text { subject to : } \\
\frac{1}{2} \sum_{i=1}^{M} A_{i} \ell_{i}\left[\frac{\sigma_{i}^{2}}{\tilde{E}_{i}}+\sum_{\beta=1}^{N_{i}} E_{i}^{\beta}\left(\varepsilon_{i}^{\beta}\right)^{2}\right]- \\
\alpha \sum_{\gamma=1}^{N} p_{\gamma} u_{\gamma}+\Pi \leq 0 \\
A_{i} \ell_{i}\left(-\varepsilon_{i}^{\beta}-\underline{\varepsilon}_{i}^{\beta}\right) \leq 0, \quad \forall i ; \forall \beta \\
A_{i}\left[\sum_{i=1}^{N} D_{i \gamma} u_{\gamma}-\left(\frac{\sigma_{i}}{\tilde{E}_{i}}+\sum_{\beta=1}^{N_{i}} \varepsilon_{i}^{\beta}\right) \ell_{i}\right]=0, \forall i .
\end{array}\right.
$$

Additional symbols appearing here are $A_{i}$ and $\ell_{i}$ for member area and length, $p_{\gamma}$ and $u_{\gamma}$ for system loads and displacement fields, $D_{i \gamma}$ for the compatibility matrix; $M$ represents the number of truss members in the system, $N$ identifies the number of independent nodal displacements $u_{\gamma}$, and $N_{i}$ is the count of independent constituents in the $i$-th member.

Formulation [P] describes a convex, constrained nonlinear programming problem. Thus the "necessary conditions" for the problem suffice to determine a unique solution, and it may be verified that the equilibrium state of the truss is given by this solution. Towards a verification of this, the Kurash-Kuhn-Tucker conditions for the problem are to be interpreted. With multipliers $\Lambda, \mu_{i}^{\beta}, v_{i}^{\beta}$ and $\kappa_{i}$ associated with constraints (C1-3), respectively, stationarity with respect to load parameter $\alpha$, and fields $\sigma_{i}, \varepsilon_{i}^{\beta}$, and $u_{\gamma}$ are (in order and after simplification)

$$
\begin{aligned}
& 1-\Lambda\left(\sum_{\gamma=1}^{N} p_{\gamma} u_{\gamma}\right)=0, \\
& \kappa_{k}=\Lambda \sigma_{k}, \quad \forall k, \\
& \Lambda E_{k}^{\beta} \varepsilon_{k}^{\beta}+\mu_{k}^{\beta}-v_{k}^{\beta}-\kappa_{k}=0, \quad \forall k ; \forall \beta, \\
& -\Lambda \alpha p_{\delta}+\sum_{i=1}^{M} A_{i} \kappa_{i} D_{i \delta}=0, \quad \forall \delta .
\end{aligned}
$$

Equation (3) simply provides for the evaluation of $\Lambda$. Substitution of $\kappa_{i}=\Lambda \sigma_{i}$ from (4) into (6) produces a statement of the system equilibrium equations in terms of stresses $\sigma_{i}$, i.e.

$\sum_{i=1}^{M} A_{i} \sigma_{i} D_{i \delta}-\alpha p_{\delta}=0, \quad \forall \delta$.

With the elimination of $\kappa_{i}$ from (5), the constituent strains are expressed as

$\varepsilon_{i}^{\beta}=\left[\sigma_{i}-\left(\tilde{\mu}_{i}^{\beta}-\tilde{v}_{i}^{\beta}\right)\right] / E_{i}^{\beta}$.

For convenience $\tilde{\mu}_{i}^{\beta}=\mu_{i}^{\beta} / \Lambda$ and $\tilde{v}_{i}^{\beta}=v_{i}^{\beta} / \Lambda$ are introduced. The solution to problem $[\mathbf{P}]$ is required to satisfy the following additional necessary (KKT) conditions:

$\Lambda\left\{\frac{1}{2} \sum_{i=1}^{M} A_{i} \ell_{i}\left[\frac{\sigma_{i}^{2}}{\tilde{E}_{i}}+\sum_{\beta} E_{i}^{\beta}\left(\varepsilon_{i}^{\beta}\right)^{2}\right]-\alpha \sum_{\gamma=1}^{N} p_{\gamma} u_{\gamma}+\Pi\right\} \leq 0$, 
$\Lambda \geq 0$,

$A_{i} \ell_{i} \tilde{\mu}_{i}^{\beta}\left(\varepsilon_{i}^{\beta}-\bar{\varepsilon}_{i}^{\beta}\right)=0, \quad \tilde{\mu}_{i}^{\beta} \geq 0$,

$A_{i} \ell_{i} \tilde{v}_{i}^{\beta}\left(-\varepsilon_{i}^{\beta}-\varepsilon_{i}^{\beta}\right)=0, \quad \tilde{v}_{i}^{\beta} \geq 0, \quad \forall i ; \forall \beta$,

$\kappa_{i} A_{i}\left[\sum_{\gamma=1}^{N} D_{i \gamma} u_{\gamma}-\left(\frac{\sigma_{i}}{\tilde{E}_{i}}+\sum_{\beta=1}^{N_{i}} \varepsilon_{i}^{\beta}\right) \ell_{i}\right]=0$.

To complete the interpretation of this system, note from (4) and (11) that $\sigma_{i} \neq 0$ implies satisfaction for the respective truss member of the strain-displacement constraint (C3). This property together with (7) assures that fields $\sigma_{i}$ and $\varepsilon_{i}^{\beta}$ in combination satisfy the system equilibrium and compatibility relations. We note that these results apply independently of the degree of material stiffening associated with the particular solution. This latter condition is interpreted uniquely for each constituent field $\varepsilon_{i}^{\beta}$ as follows. If $0 \leq \varepsilon_{i}^{\beta}<\bar{\varepsilon}_{i}^{\beta}$ or $0 \leq-\varepsilon_{i}^{\beta}<\varepsilon_{i}^{\beta}$, from (10) $\tilde{\mu}_{i}^{\beta}=0$ and $\tilde{v}_{i}^{\beta}=0$, whereby (5) provides (for the $\beta$-th constituent)

$\varepsilon_{i}^{\beta}=\frac{\sigma_{i}}{E_{i}^{\beta}}$.

In other words, the constituents for which this relation holds follow their respective linear stress-strain behaviour. Otherwise, for $\tilde{\mu}_{i}^{\beta}>0$ or $\left(\tilde{v}_{i}^{\beta}>0\right.$ ), from (10) $\varepsilon_{i}^{\beta}-\bar{\varepsilon}_{i}^{\beta}=0$ (or $-\varepsilon_{i}^{\beta}-\varepsilon_{i}^{\beta}=0$ ), i.e. the constituent is in the locked state. Accordingly, multipliers $\tilde{\mu}_{i}^{\beta}$ and $\tilde{v}_{i}^{\beta}$ may be evaluated from (5) as

$\tilde{\mu}_{i}^{\beta}=\sigma_{i}-E_{i}^{\beta} \bar{\varepsilon}_{i}^{\beta}, \quad \forall i ; \forall \beta$,

$\tilde{v}_{i}^{\beta}=-\sigma_{i}-E_{i}^{\beta} \underline{\varepsilon}_{i}^{\beta}$.

One or the other of these conditions necessarily applies to every constituent, i.e. for each value of $\beta$ and over all truss elements. It may be verified from the necessary conditions that for every element $i$, each constituent $\beta$ within the set

$B_{i}=\left\{\beta \mid\left(\beta=1,2, \ldots, N_{i}\right)\right\}$ belongs either to

$S_{i}=\left\{\beta \in B_{i} \mid\left(\varepsilon_{i}^{\beta}<\bar{\varepsilon}_{i}^{\beta}\right)\right.$ and $\left.\left(-\varepsilon_{i}^{\beta}<\underline{\varepsilon}_{i}^{\beta}\right)\right\}$,

$\bar{S}_{i}=\left\{\beta \in B_{i} \mid\left(\varepsilon_{i}^{\beta}=\bar{\varepsilon}_{i}^{\beta}\right)\right\}$, or

$\underline{S}_{i}=\left\{\beta \in B_{i} \mid\left(-\varepsilon_{i}^{\beta}=\underline{\varepsilon}_{i}^{\beta}\right)\right\}$, and $B_{i}=S_{i} \cup \bar{S}_{i} \cup \underline{S}_{i}$.

In this way it is clear that the constituent fields $\varepsilon_{i}^{\beta}$ are fully determined via the necessary conditions listed, and this together with the already indicated solution $\sigma_{i}$ provides that the unique solution to the global equilibrium analysis problem $[P]$ is complete.

In summary, the means has been described by which the equilibrium analysis problem for arbitrary trusses, having members whose constitutive properties correspond to a parameter-specified polygonal form of stiffening material, is represented in the form of the convex problem $[\mathbf{P}]$. It may be of interest to note that this model is dual in concept to a formulation presented earlier by Taylor (1993) for softening materials.

\subsection{Computational examples}

The following examples demonstrate the feasibility and utility of using the model $[P]$ for equilibrium analysis to predict computationally the evolution of response of truss structures composed of nonlinear materials. The model was implemented using a commercially available constrained optimizer program incorporated in MATLAB (1992), running on a Hewlett-Packard computer. This program is based on a sequential quadratic programming algorithm (Schittkowski 1985). The model was interpreted directly into the program, with no special provisions made to improve the efficiency of the solution procedure. For instance, the gradients were calculated numerically rather than provided in analytic form. For all the models discussed here, each solution required on the order of $10^{3}$ seconds on the computer.

The numerical implementation was checked by comparing simple one- and two-dimensional trusses with a typical analysis pieced together manually. However a special feature of this model is that equilibrium is not explicitly specified, i.e. equilibrium is a consequence of the minimization. Thus verification that the end result truly satisfies equilibrium provides a check on the numerical implementation of the minimization.

\subsubsection{Pentagonal truss}

The first model demonstrates the application of this method of analysis to a typical truss geometry. This geometry consists of five nodes and nine bar elements as shown in Fig. 3 . The truss is loaded at node number 1 by a load inclined at an angle of $\pi / 5$ from the horizontal. Nodes 4 and 5 are constrained not to move. Each truss member has the same material and sectional properties. The material consists of a strictly linear constituent and two symmetric stiffening constituents. That is, $\tilde{E}_{i}=128, E_{i}^{1}=256, E_{i}^{2}=64, \bar{\varepsilon}_{i}^{1}=\underline{\varepsilon}_{i}^{1}=$ $0.003, \bar{\varepsilon}_{i}^{2}=\varepsilon_{i}^{2}=0.006$, and $A_{i}=0.1$ for $\{i \in 1,2, \ldots, 9\}$.

The analysis for given values of energy bound $(\Pi)$, and load and material data proceeds by iteration within the program on the load factor $(\alpha)$, displacement $\left(u_{i}\right)$, and constituent $(\sigma),\left(\varepsilon_{i}^{\beta}\right)$ variables until the load factor is sufficiently converged toward a minimum. The loads at each specified energy resulting from this minimization are shown in Fig. 4. The energy parameter is approximately quadratically related to the load factor. The energy load relationship is not truly quadratic because of the nonlinear constitutive response; it is in fact piecewise quadratic in each region where the active constraints are the same. (This piecewise behaviour is not evident on the scale of this figure.)

The displacement of the unconstrained nodes (1,2 and 3$)$ as a function of the load are given in Fig. 5 . Here the nonlinear behaviour inherent in the model is clearly evident. At small loads each truss element has an effective modulus, $E_{i}$, given by (15), which is indicative of the series construction of the constituents.

$\frac{1}{E_{i}}=\frac{1}{\tilde{E}_{i}}+\sum_{\beta=1}^{2} \frac{1}{E_{i}^{\beta}}$.

As the allowable energy $\Pi$ is increased, and with the remaining data held fixed, certain constituents reach their constraint values. A constituent that has an active constraint is "locked" in the sense that the constituent can no longer 


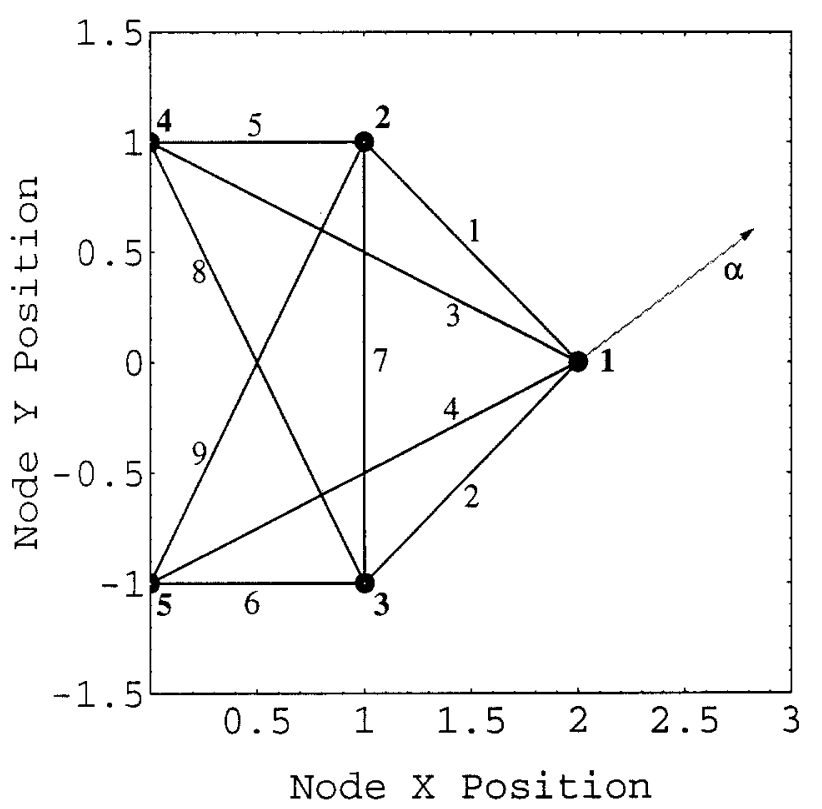

Fig. 3. Pentagonal truss geometry and load vector

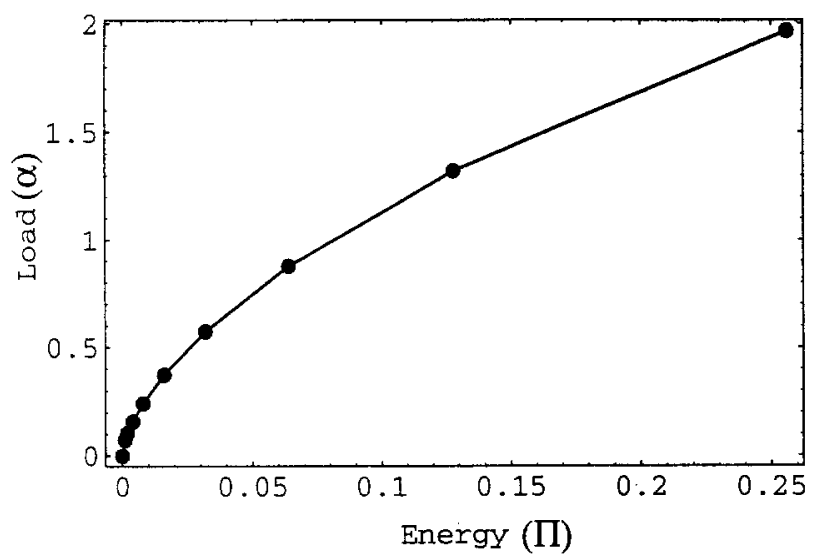

Fig. 4. Load vs. specified energy

deform. In effect, the modulus of the constituent becomes infinite and its inverse becomes zero in (15), thereby increasing the effective modulus of the element. When all the constituents for an element become locked, the tangential modulus of the subsequent response becomes the modulus of the linear constituent, i.e. $E_{i}=\tilde{E}_{i}$.

The stress distribution amongst the truss elements, with the specified energy as a parameter, is displayed in Fig. 6. At this scale, the effect of the nonlinear behaviour is not overtly evident. The nonlinear behaviour is shown clearly, however, in a plot of the stress or strain as a function of the load. The strain of the first constituent for each member, depicted as a function of the load is given in Fig. 7.

At sufficiently small energies (and loads) the system behaves linearly. However, as soon as one of the constituents "locks", nonlinear behaviour ensues. The evolution of the first hardening constituent, $\varepsilon_{i}^{1}$, is representative of the behaviour of all the constituents for the remaining elements (i.e. $\sigma_{i}$, and $\varepsilon_{i}^{2}$ ), except note that the linear constituent, $\sigma_{i}$, is not limited. This type of nonlinear behaviour is exceptional in the sense that the usual ingrained notions of proportional

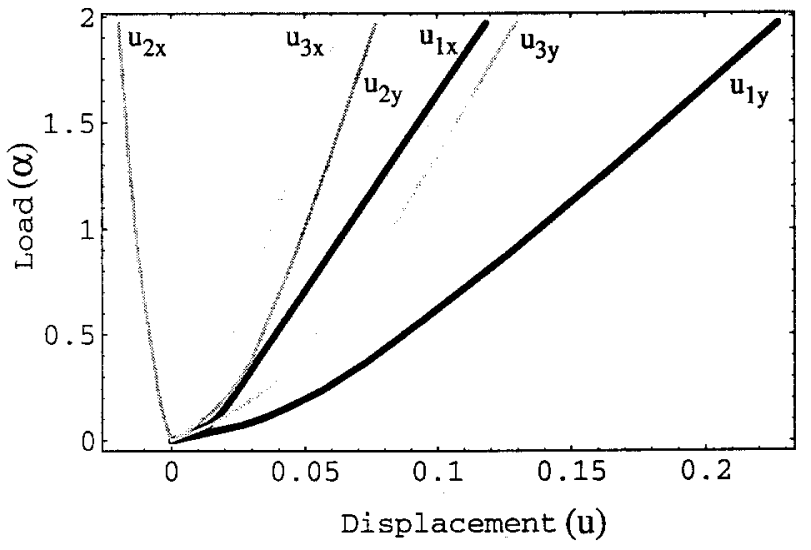

Fig. 5. Load vs. displacement

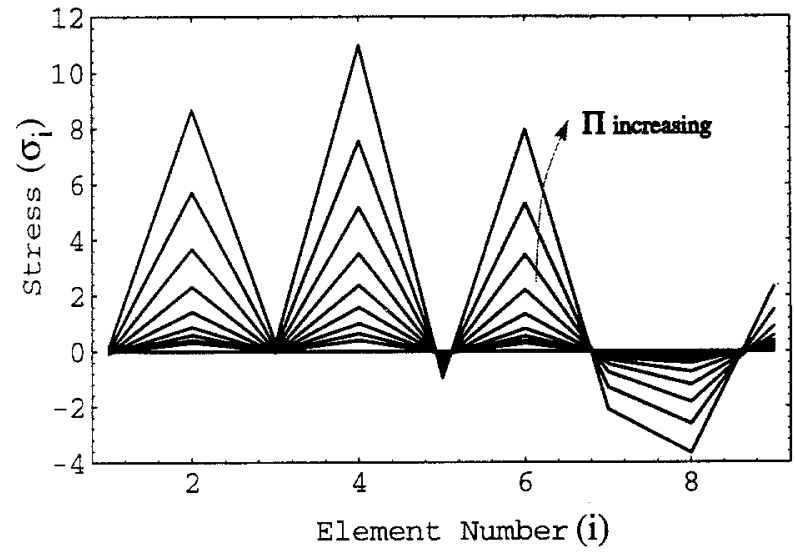

Fig. 6. Stress at each element for each energy point displayed in Fig. 4. Note that the specified energy values are a separated by a factor of 2

loading are not followed.

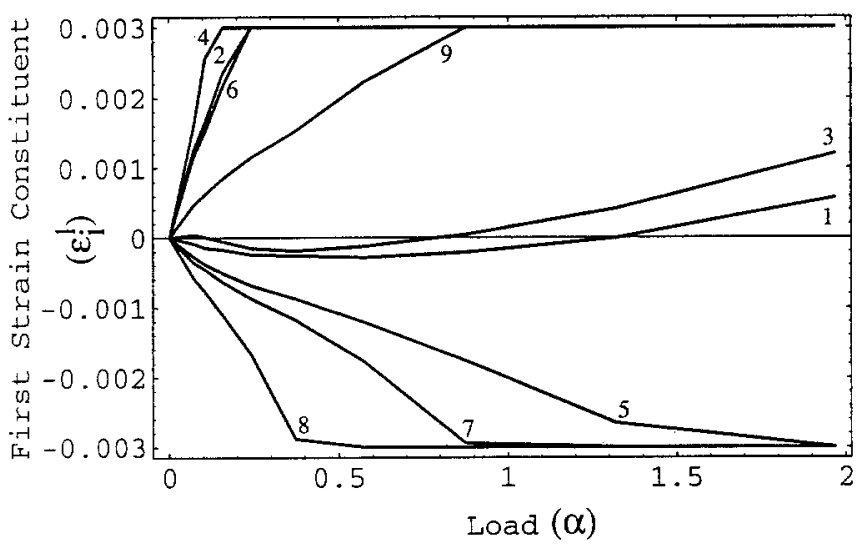

Fig. 7. Strain of the first constituent, for each of the nine elements, as a function of load

\subsubsection{Pyramidal truss}

The preceding example demonstrates that this technique can be employed to analyse a typical truss geometry with an atypical nonlinear hardening constitutive law. However, in many respects the example is cumbersome in that the evolution of behaviour in the constituents is not immediately obvious. Part of this stems from a geometry with elements that are intertwined at disparate angles. Interpreting these results 
requires a ponderous process of piecing together aspects of several of the figures. To simplify matters, especially for the subsequent example design problem, a simpler geometry was explored.

The second example problem demonstrates the application of this method of analysis to a simpler truss geometry, one for which the nonlinear evolution is easier to follow. This geometry consists of twelve nodes and eleven bar elements as shown in Fig. 8. The truss is loaded at node number 1 by a horizontal load $\alpha$. All nodes except the first are constrained not to move, i.e. representing a fixed support. Paralleling the previous example, each element has the same unbounded linear constituent modulus, the same cross-sectional area and is composed of two symmetrically stiffening constituents. That is, $\tilde{E}_{i}=64, \bar{\varepsilon}_{i}^{1}=\varepsilon_{i}^{1}=0.008, \bar{\varepsilon}_{i}^{2}=\underline{\varepsilon}_{i}^{2}=0.016$, and $A_{i}=$ 0.1 for $\{i \in 1,2, \ldots, 11\}$. However, here the moduli for the hardening constituents vary over the truss. This distribution is shown in Fig. 9. The moduli have been chosen such that - $E_{i}^{1}+E_{i}^{2}=160$, so that for small energies where the system is linear, the geometry, material properties and loading are all symmetric. However, here the locking of a constituent will not only cause the system to become nonlinear, but to lose symmetry as well.

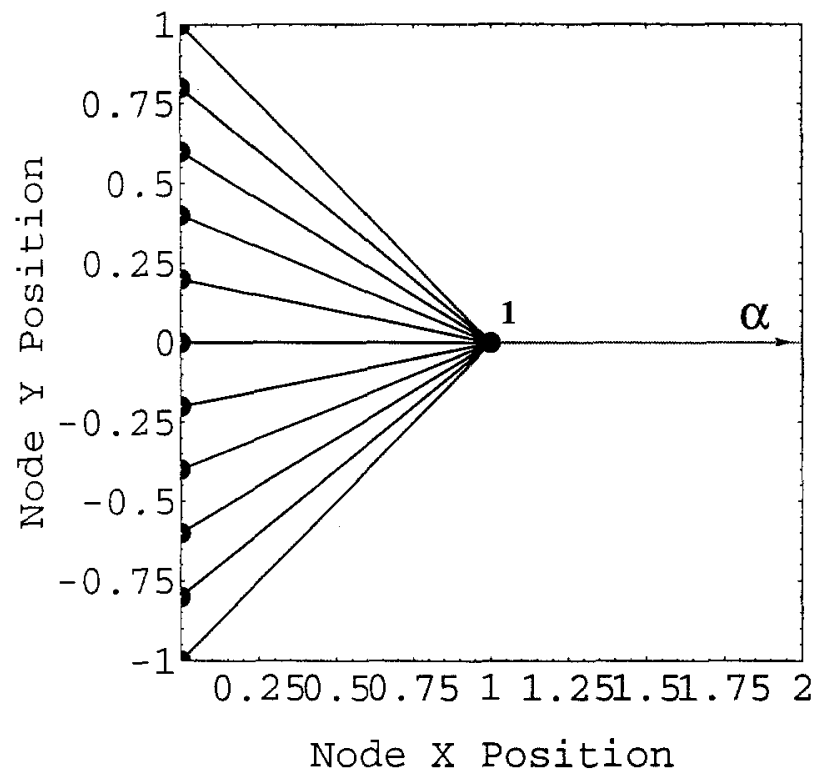

Fig. 8. Pyramidal truss geometry and symmetric loading

Thus, as shown in Fig. 10, the deformation of the vertex of the truss in the $y$-direction $\left(u_{y}\right)$ is initially zero, owing to the symmetry of the problem. As the material evolves, the symmetry is lost and there is a nonzero $\left(u_{y}\right)$ deformation. At higher energies and loads, all the constituents lock, and the material response again becomes symmetric and the deformation in the $y$-direction approaches zero.

The evolution of the linear constituents $\left(\sigma_{i}\right)$ is shown in Fig. 11. Evolutions of the two locking constituents $\left(\varepsilon_{i}^{1}\right.$ and $\varepsilon_{i}^{2}$ ) are shown in Figs. 12 and 13 , respectively.

These figures reinforce the statements concerning the load-displacement behaviour of the system. At small energies and loads, the system remains linearly elastic. At higher energies, the locking constituents reach their constraints. This

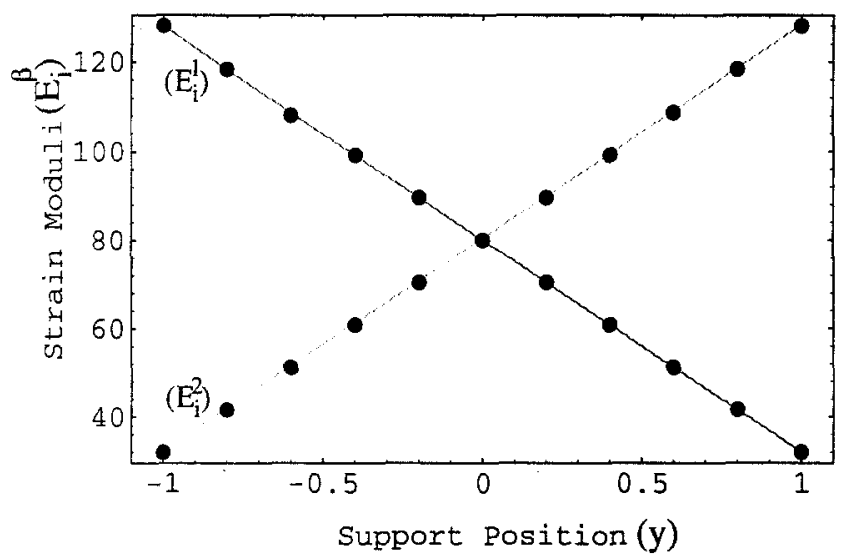

Fig. 9. Moduli of the "locking" constituents as a function of member support position at $x=0$

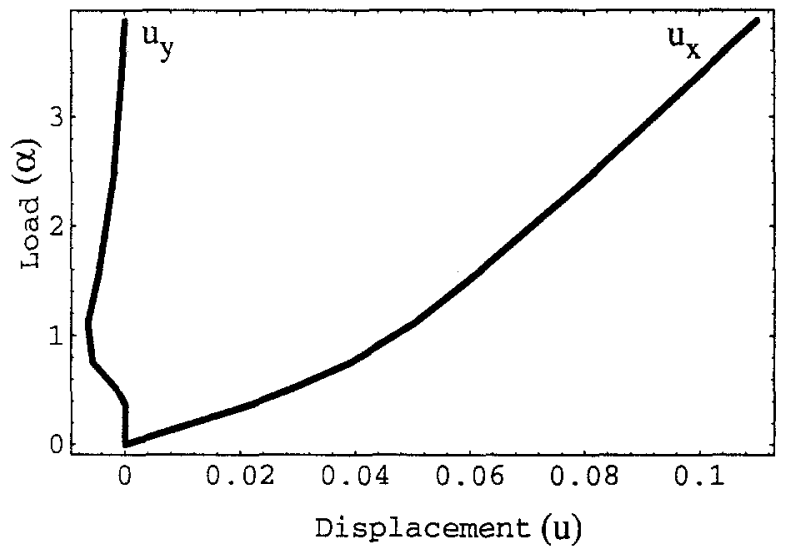

Fig. 10. Load vs. displacement of the free node

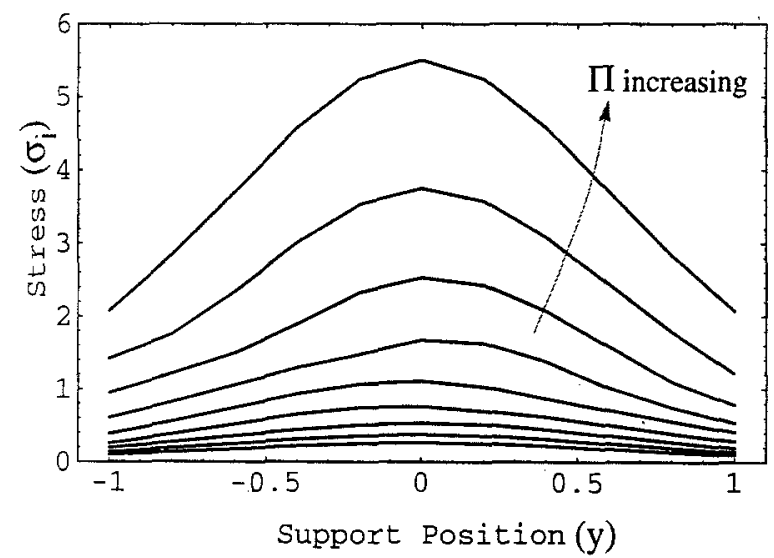

Fig. 11. Stress of the enduring constituents as a function of support position with increasing energy as a parameter

causes the nonlinearity in the load-displacement behaviour. This is evident in the enduring constituent $\left(\sigma_{i}\right)$ as a slight asymmetry in the stress distribution. At higher energies, when almost all of the members have "locked", the stress distribution has nearly recovered its symmetry.

\section{Design of material properties for the optimum truss}

A formulation is presented in this section that relates to the prediction of optimal material properties in the context of a 


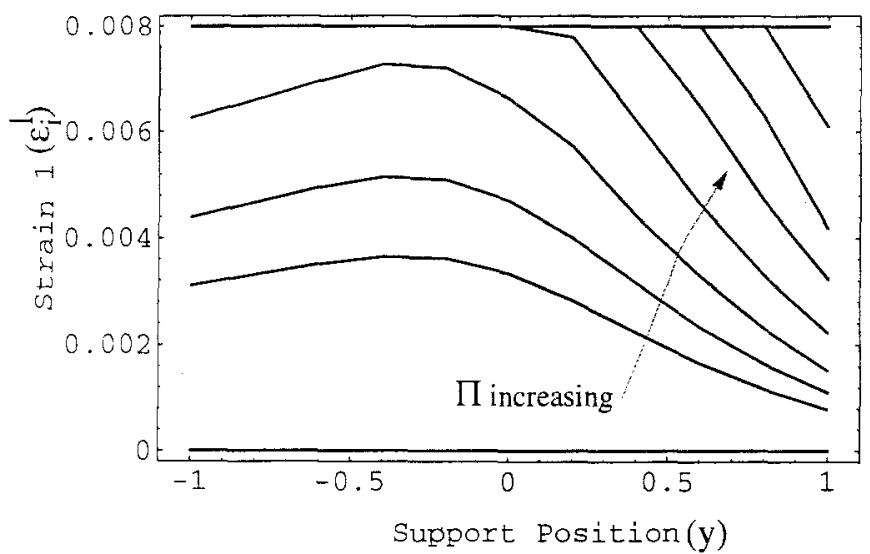

Fig. 12. Strain in the first locking constituent as a function of support position with increasing energy as a parameter

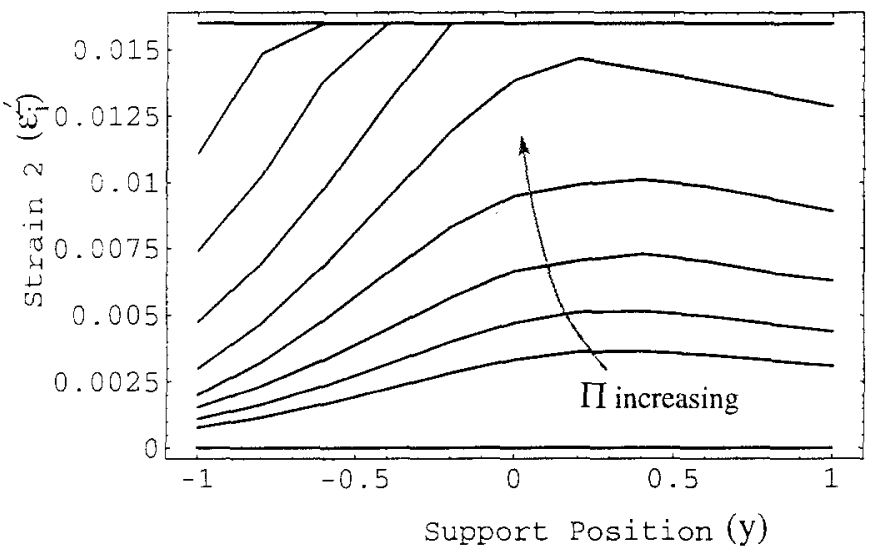

Fig. 13. Strain in the second locking constituent as a function of support position with increasing energy as a parameter

global structural design problem. Specifically, the purpose is to determine the distribution of adjustments to the material properties identified with locking limits, for all or a part of the set of constituents, so that a lower bound on the overall structural "energy" is maximized. This is equivalent to minimizing an equilibrium state for which the total energy is no less than a specified value. (The two formulations are alternate statements of equivalent, isoperimetric problems.) Stated differently, the optimal adjustments are those for which stiffening of the structure is deferred as much as possible. The optimal design problem presented here is a convex problem stated in isoperimetric form. An interpretation is given for the "necessary condition" of this problem and the optimal quality of the solution to the system is verified.

\subsection{Design problem formulation}

Modifications to the locking limits are symbolized by vectors $\phi_{i}^{\beta}$ and $\psi_{i}^{\beta}$, which represent, respectively, the changes in value of the upper and lower bounds on constituent strains. Components of these vectors are restricted to be non-negative, and the overall modification to structural material is limited by the isoperimetric constraint

$\sum_{\beta} \sum_{i} A_{i} \ell_{i}\left(\phi_{i}^{\beta}+\psi_{i}^{\beta}\right)-R \leq 0$.

Bound $R$ appearing in this constraint is a measure of total resource for material modification and is a new element of data. The design problem is described formally relative to the analysis problem $[\mathbf{P}]$, in terms of an outer " $\mathbf{m i n}$ " with respect to the design parameters $\phi_{i}^{\beta}$ and $\psi_{i}^{\beta}$. In other words, the sets of design parameters are to be determined according to

$$
[\mathbf{M}]\left\{\begin{array}{l}
\min _{\varphi, \psi}\left[\min _{\alpha, \sigma, \varepsilon, u} \alpha\right] \\
\text { subject to: } \\
\frac{1}{2}\left\{\sum_{i=1}^{M} A_{i} \ell_{i}\left[\frac{\sigma_{i}^{2}}{\tilde{E}_{i}}+\sum_{\beta=1}^{N_{i}} E_{i}^{\beta}\left(\varepsilon_{i}^{\beta}\right)^{2}\right]\right\}- \\
\alpha \sum_{\gamma=1}^{N} p_{\gamma} u_{\gamma}+\Pi \leq 0 \\
A_{i} \ell_{i}\left[\varepsilon_{i}^{\beta}-\left(\bar{\varepsilon}_{i}^{\beta}+\phi_{i}^{\beta}\right)\right] \leq 0 \\
A_{i} \ell_{i}\left[-\varepsilon_{i}^{\beta}-\left(\underline{\varepsilon}_{i}^{\beta}+\psi_{i}^{\beta}\right)\right] \leq 0 \\
A_{i}\left[\sum_{\gamma=1}^{N} D_{i \gamma} u_{\gamma}-\left(\frac{\sigma_{i}}{\tilde{E}_{i}}+\sum_{i=1}^{N} \varepsilon_{i}^{\beta}\right) \ell_{i}\right]=0 \\
-\phi_{i}^{\beta} A_{i} \ell_{i} \leq 0, \\
-\psi_{i}^{\beta} A_{i} \ell_{i} \leq 0, \quad \forall i ; \forall \beta, \\
N_{i} \sum_{i}^{M} A_{i} \ell_{i}\left(\phi_{i}^{\beta}+\psi_{i}^{\beta}\right)-R \leq 0 \\
\sum_{\beta=1}^{\beta} \sum_{i=1}^{\beta},
\end{array}\right.
$$

The order of the "min"s is interchangeable [see e.g. Jog et al. (1993) and Bendsøe et al. (1992) for a justification], and so it is justifiable to consider separately the necessary conditions associated with the design part of the problem [M]. With the introduction of $\eta_{i}^{\beta}, \zeta_{i}^{\beta}$, and $\Gamma$ as multipliers associated with the last three amongst the constraints listed, i.e. the constraints associated with design variables $\phi_{i}^{\beta}$ and $\psi_{i}^{\beta}$, these conditions are

$-\mu_{i}^{\beta}-\eta_{i}^{\beta}+\Gamma=0, \quad \forall i ; \forall \beta$

$-v_{i}^{\beta}-\zeta_{i}^{\beta}+\Gamma=0$.

It may be verified that so long as $R>0$ in problem [M], the multiplier $\Gamma$ satisfies $\Gamma>0$. An immediate consequence of (17) is that in order for a modification of the locking limit to occur, it is necessary that the respective strain constituent should have become locked in the original, i.e. unmodified system. This result may be verified via the following argument. Suppose that constraint (C2.1) of problem [M] is met by inequality; then the associated multiplier $\mu_{i}^{\beta}$ has value zero, and so from (17)

$\eta_{i}^{\beta}=\Gamma, \quad \forall i ; \forall \beta$.

Thus in view of the (Kurash-Kuhn-Tucker) requirement $\eta_{i}^{\beta} \phi_{i}^{\beta}=0 \forall i ; \beta$, it follows that, for components governed by (19), satisfaction of constraint (C2.1) by inequality implies $\phi_{i}^{\beta}=0$, i.e. the associated locking constraint bound value is not modified. Similar argument leads to the counterpart of this result for the constraint bounding strain on the negative side, and completes the proof.

To continue the interpretation, note that for $\mu_{i}^{\beta}>0$, from constraint $(\mathrm{C} 2.1)$ of problem $[\mathrm{M}], \varepsilon_{i}^{\beta}-\left(\bar{\varepsilon}_{i}^{\beta}+\phi_{i}^{\beta}\right)=0$. It follows from (C2.2) that in this case $v_{i}^{\beta}=0$. Given the similar argument as it applies in reverse, i.e. $v_{i}^{\beta}>0$ implies 
$\mu_{i}^{\beta}=0$, we have that the multipliers $\mu_{i}^{\beta}$ and $v_{i}^{\beta}$ are orthogonal; $\mu_{i}^{\beta} v_{i}^{\beta}=0$ for all $i$ and $\beta$. The alternative possible consequences of the necessary conditions (17) and (18) are considered next. As noted from $(\mathrm{C} 2.1)$ in the case $\mu_{i}^{\beta}>0$ \{or from $(\mathrm{C} 2.2)$ for $v_{i}^{\beta}>0$ \} that

$\varepsilon_{i}^{\beta}-\left(\bar{\varepsilon}_{i}^{\beta}+\phi_{i}^{\beta}\right)=0$,

$\left\{\right.$ or $\left.-\varepsilon_{i}^{\beta}-\left(\underline{\varepsilon}_{i}^{\beta}+\psi_{i}^{\beta}\right)=0\right\}$.

If also $\eta_{i}^{\beta}>0$ or $\left\{\right.$ or $\left.\zeta_{i}^{\beta}>0\right\}$, then there is no modification to the strain bound i.e. $\phi_{i}^{\beta}=0\left\{\right.$ or $\left.\psi_{i}^{\beta}=0\right\}$, and so the value of the associated strain constituent is just equal to the original locking strain bound, $\varepsilon_{i}^{\beta}=\bar{\varepsilon}_{i}^{\beta}$ or $\left.-\varepsilon_{i}^{\beta}=\underline{\varepsilon}_{i}^{\beta}\right\}$. More generally one expects to find $\phi_{i}^{\beta}>0$ in conjunction with $\mu_{i}^{\beta}>0$ \{or $\psi_{i}^{\beta}>0$ with $\left.v_{i}^{\beta}>0\right\}$, and then the associated (optimal) modifications are evaluated directly from (20) and (21). Referring to expressions for stress/strain relations obtained in Section 2, and with the substitution of $\mu_{i}^{\beta}=\Gamma$ or $v_{i}^{\beta}=\Gamma$ from the results above, the stresses evaluated according to the solution for the optimally modified locking constraint values are given by

$\sigma_{i}-E_{i}^{\beta}\left(\bar{\varepsilon}_{i}^{\beta}+\phi_{i}^{\beta}\right)=\Gamma / \Lambda$ where $\mu_{i}^{\beta}>0$,

$-\sigma_{i}-E_{i}^{\beta}\left(\underline{\varepsilon}_{i}^{\beta}+\psi_{i}^{\beta}\right)=\Gamma / \Lambda$ where $v_{i}^{\beta}>0$.

According to these results the difference between total stress $\sigma_{i}$ and the values of stress at with locking occurs ("locking stress") has constant value. This remarkable result holds for all constituent strains $\varepsilon_{i}^{\beta}$ (over the structural system) which have become locked at the respective loads. Also, as a sidelight, it may be observed from this property of the results that the solution for design problem $[\mathbf{M}]$ minimizes the maximum measure of the cited difference in stress values (again, over the entire structure).

\subsection{Computational examples}

The above design problem was applied to the same pyramidal truss that was treated in Section 2.2.2 as the second example of analysis. As noted in the problem description given above, the evolution of local stiffening in a structure made of stiffening material may be determined from the results of a set of solutions to the original analysis problem. The end result of a series of such solutions is summarized in Fig. 14 with the details of one of the locking constituents shown in Fig. 15. The overall effect of the design is, as expected, to minimize the structural stiffness of the truss. Here this minimization predicts a modification of stiffening limits that has structural response tend toward that of the linear structure.

At small energies, there is no modification to the load distribution in the problem. As the specified energy is increased, the locking constraints are modified to allow the system to remain linear elastic (i.e. not locked). The distribution of the locking parameter varies as a function of energy. Considering that the extension of the modelling for this problem to accommodate the present purpose, i.e. the prediction of optimal relaxation of the locking bounds, it is understandable that the solution to the design problem varies with load (or

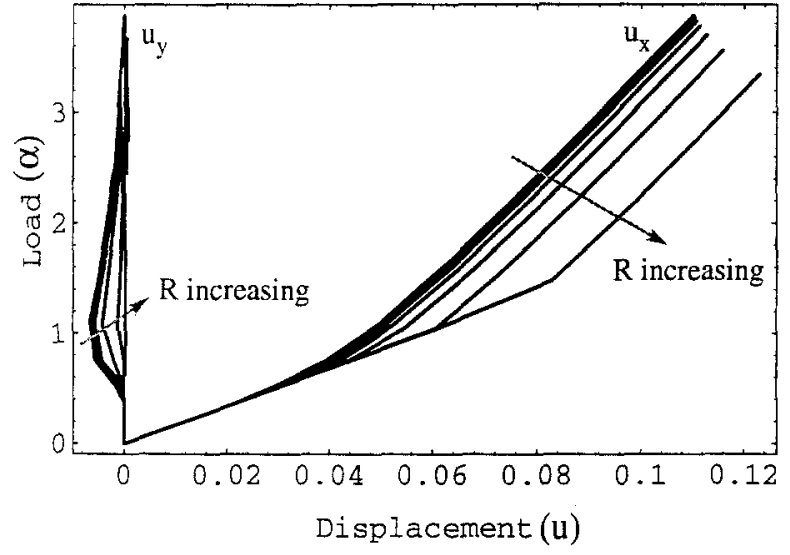

Fig. 14. Load versus displacement for increasing values of resource of the locking constraint

energy) level. This is characteristic of problems in optimal design in the presence of a constitutive nonlinearity.

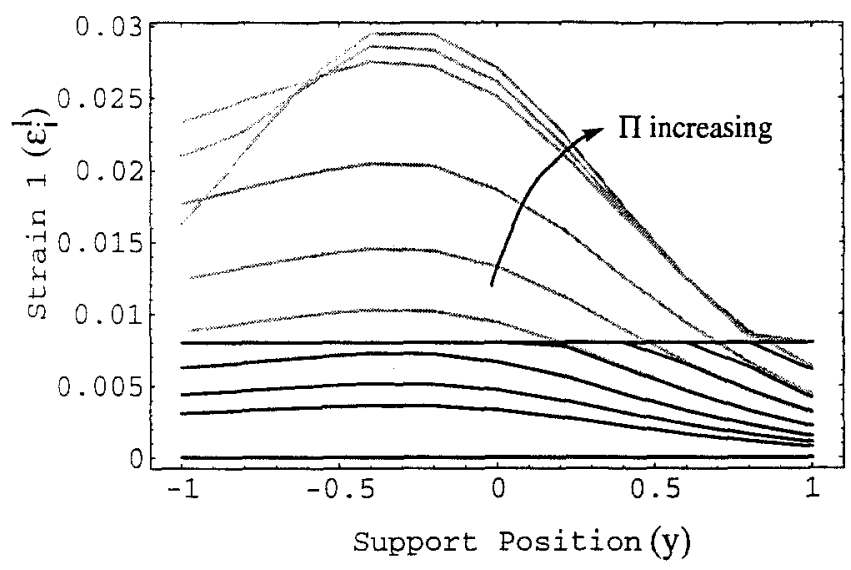

Fig. 15. Effect of available resource on the strain distribution, and limits for the first strain constituent. The grey lines indicate the improved design, the black lines indicate the original design (i.e. Fig. 12)

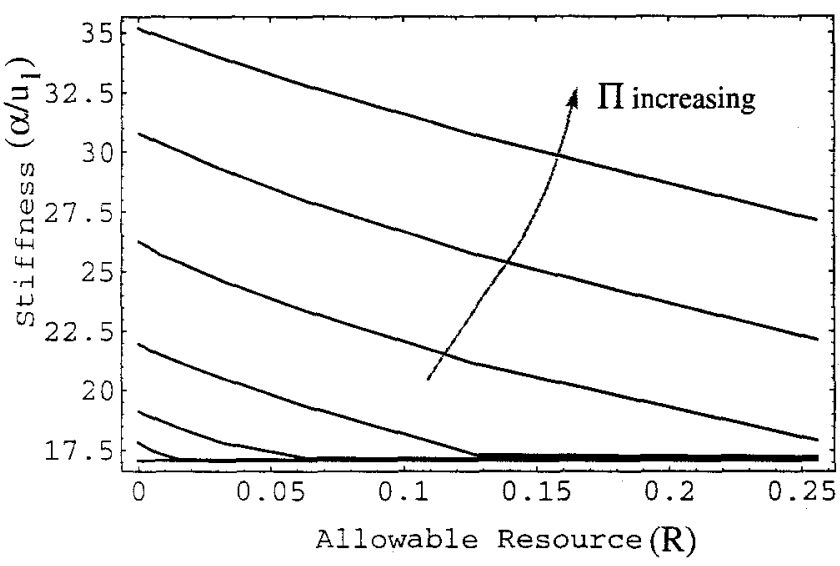

Fig. 16. Overall stiffness of the structure for increasing values of allowable resource and increasing energy

It may be established as well from the results for the design problem described in this section, that there exists an upper limit say $\bar{R}$, such that the problem admits sensible interpretation for values of modification resource $R$ lying 
within the range $0<R<\bar{R}$. The upper bound value, which varies with overall load on the structure, is the value such that in the structure with optimally modified material, all constituent strains $\varepsilon_{i}^{\beta}$ will at most have just reached their respective locking limits in the fully loaded system. In other words, the solution associated with $R=\bar{R}$ provides that no locking occurs as the loads are applied. As a consequence, the structural response is simply linear. Note that in this case the conventional measure of compliance is minimized for the optimal structure. This behaviour is demonstrated in Fig. 16.

\section{Concluding remarks}

The analysis of a truss system composed of a nonlinear material has been demonstrated using a mixed form equilibrium principle. This principle was extended to incorporate the design of parameters identified with the constituent locking limits of the stiffening material. Perhaps the most significant conceptual steps are in the formulation of the original principle described in the analysis problem $[\mathbf{P}]$. Here a set of constituents is assembled to provide an overall nonlinear material response. This formulation leaves normal conservation statements (e.g. energy, compatibility, and constituent limits) in their simple form as constraints on the response of the system. For instance, although the overall response of the system is generally nonlinear, the mathematics is remarkably tidy. This form of the analysis problem allows the straightforward insertion of the parameters, and simplifies extension of the extremum problem statement to cover the design of the material properties. One can speculate that other aspects of $[\mathbf{P}]$ (e.g. the infinitesimal of the strain-displacement equations) might also be directly extended (e.g. finite straindisplacement equations).

\section{References}

Arthurs, A.M. 1980: Complementary variational principles (2nd edition). Oxford: Clarendon Press

Bendsøe, M.P.; Guedes, J.M; Haber, R.B.; Pedersen, P.; Taylor,
J.E. 1992: An analytical model to predict optimal material properties in the context of optimal structural design. J. Appl. Mech. (to appear)

Comi, C.; Corigliano, A.; Maier, G. 1991: Extremum properties of finite step solutions in elastoplasticity with nonlinear mixed hardening. Int. J. Solids \& Struct. 27, 965-981

Hewlett-Packard Computer Model 9000/715

Hlavacek, I.; Rosenberg, J.; Beagles, A.E.; Whiteman, J.R. 1992: Variational inequality formulation in strain space and finite element solution of an elasto-plastic problem with hardening. Comput. Meth. Appl. Mech. Engrg. 94, 93-112

Jog, C.; Haber, R.B.; Bendsøe, M.P. 1993: Topology design with optimized, self adaptive materials. DCAMM Report No. 457, The Danish Center for Applied Mathematics and Mechanics, The Technical University of Denmark

The Math Works, Inc. 1992: MATLAB Version 4.0a, Natick, MA

Oden, J.T.; Reddy, J.N. 1976: Variational methods in theoretical mechanics. Berlin, Heidelberg, New York: Springer

Prager, W. 1957: On ideal locking materials. Trans. Soc. Rehology 1, 169-175

Schittkowski, K. 1985: NLQPL: a Fortran subroutine solving constrained nonlinear programming problems. Operations Research $5,485-500$

Sewell, M.J. 1987: Maximum and minimum principles. Cambridge: Cambridge University Press

Simo, J.C.; Kennedy, J.G.; Taylor, R.L. 1989: Complementary mixed finite element formulations for elastoplasticity. Comput. Meth. Appl. Mech. Engrg. 74, 177-206

Taylor, J.E. 1992: A global extremum principle in mixed form for equilibrium analysis with elastic/hardening materials (a generalized minimum potential energy principle). J. Appl. Mech. (to appear)

Taylor, J.E. 1993: A global extremum principle for the analysis of solids composed of softening material. Int. J. Solids \& Struct. 30, 2057-2069

Washizu, K. 1982: Variational methods in elasticity and plasticity. New York: Pergamon Press 